\title{
Introduction to the special issue: project scheduling under uncertainty
}

\author{
Erik Demeulemeester · Willy Herroelen
}

Published online: 11 May 2007

(C) Springer Science+Business Media, LLC 2007

The vast majority of the research efforts in project scheduling over the past several years have concentrated on the development of exact and heuristic procedures for the generation of a workable baseline schedule (pre-schedule or predictive schedule) assuming complete information and a static and deterministic environment. Most often the baseline schedule is constructed by solving the well-known deterministic resource-constrained project scheduling problem (RCPSP). This problem (problem $m, 1|c p m| C_{\max }$ in the notation of Herroelen et al. 2000) involves the determination of a baseline schedule that satisfies both the finish-start, zero-lag precedence constraints between the activities and the renewable resource constraints under the objective of minimizing the project duration (for recent comprehensive overviews of the literature, we refer to Demeulemeester and Herroelen 2002 and Neumann et al. 2003).

During execution, however, a project may be subject to considerable uncertainty, which may lead to numerous schedule disruptions. Activities can take longer or shorter than primarily expected, resource requirements or availabilities may vary, ready times and due dates may change, new activities might have to be inserted in the schedule, etc.

In this special issue we have collected six articles that examine several aspects of scheduling under uncertainty. We did not restrict the topics; they simply illustrate contemporary research in the field. The contribution of the six articles can be summarized as follows.

E. Demeulemeester $(\bowtie) \cdot$ W. Herroelen

Research Center for Operations Management, Katholieke

Universiteit Leuven, Leuven, Belgium

e-mail: erik.demeulemeester@econ.kuleuven.be

W. Herroelen

e-mail: willy.herroelen@econ.kuleuven.be
When it is worthwhile to work with the stochastic RCPSP by F. Ballestin investigates the conditions under which a stochastic project scheduling approach is to be preferred above deterministic project scheduling based on average activity durations. The author demonstrates that the use of activity based scheduling policies in combination with a sample algorithm and a genetic algorithm yield very good results with the genetic algorithms offering the best performance.

A two-stage stochastic programming approach for project scheduling with uncertain activity durations by G. Zhu, J.F. Bard and G. Yu investigates the problem of setting target times (due dates) for project activities with random durations. The objective is to balance the cost of project completion as a function of activity due dates with the expected penalty incurred by deviating from the specified values. Using two-stage integer linear stochastic programming, target times are determined in the first stage followed by the development of a detailed project schedule in the second stage. The authors draw a link between the project problem under uncertainty and the traditional newsvendor problem. An important insight resulting from their analysis is that when deviations from pre-specified target times are considered, early schedules are generally not obtained. Moreover, the optimal target completion time for a project may be greater than the makespan of all early schedules under any scenario. When crashing is permitted, traditional procedures for determining activity target times must be updated to account for uncertainty.

Resource allocation in stochastic, finite-capacity, multiproject systems through the cross entropy methodology by I. Cohen, B. Golany and A. Shtub addresses the problem of resource allocation in a finite-capacity multi-project system. The authors model the system as a queuing network that is controlled by limiting the number of concurrent projects. The authors propose a Cross Entropy (CE) 
based approach to determine near-optimal resource allocations. The approach finds the resource allocation that heuristically minimizes the projects' average throughput time.

A classification of predictive-reactive project scheduling procedures by S. Van de Vonder, E. Demeulemeester and W. Herroelen discusses the results obtained by a large experimental design set up to evaluate several predictive-reactive resource-constrained project scheduling procedures under the composite objective of maximizing both the schedule stability and the timely project completion probability when activity durations are stochastic and the project has a predefined deterministic due date. Using an exact procedure for deriving minimum makespan baseline schedules and performing a complete rescheduling upon schedule breakage excels in makespan performance but is clearly outperformed in terms of schedule stability. Using heuristic reactive procedures is computationally far less demanding and obtains comparable makespan performance at smaller stability costs. Combining an exact baseline scheduling procedure with a reactive procedure that aims at maximizing stability yields acceptable makespan and stability performance. Very promising results are obtained by proactive baseline scheduling heuristics that aim at generating stable baseline schedules.

A robust approach for the single-machine scheduling problem by C. Briand, H. Trung La and J. Erschler describes a robust approach for the single machine scheduling problem with ready times and maximum lateness objective. The method characterizes a large set of optimal solutions allowing to switch upon schedule disruption from one solution to another without performance loss. It is based on a dominance theorem that characterizes a set of dominant sequences, using the interval structure defined by the relative order of the release and due dates of the jobs. A branch and bound procedure is developed which modifies the interval structure of the problem in order to tighten the dominant set of sequences.

Scheduling for stability in single-machine production systems by R. Leus and W. Herroelen presents a model for single-machine scheduling with stability objective and a common deadline. The authors develop a branch-and-bound algorithm for solving an approximate formulation of the model. The algorithm is exact when exactly one job is disrupted during schedule execution. The model is shown to yield consistently good results for a wide range of variability settings.

\section{References}

Demeulemeester, E., \& Herroelen, W. (2002). Project scheduling-A research handbook. Boston: Kluwer Academic.

Herroelen, W., De Reyck, B., \& Demeulemeester, E. (2000). On the paper 'Resource-constrained project scheduling: notation, classification, models and methods' by Brucker et al. European Journal of Operational Research, 128, 221-230.

Neumann, K., Schwindt, C., \& Zimmermann, J. (2003). Project scheduling with time windows and scarce resources: Temporal and resource-constrained project scheduling with regular and nonregular objective functions. Heidelberg: Springer. 